\title{
Docentes de inglés de primaria, secundaria y terciaria: Sus creencias pedagógicas sobre sus estudiantes
}

\section{Primary, secondary and tertiary teachers of English: their beliefs about their learners}

\author{
Claudio Díaz-Larenas ${ }^{1}$ \\ Universidad de Concepción \\ Dirección de Docencia \\ Facultad de Educación \\ Biobio, Chile \\ claudiodiaz@udec.cl \\ Paola Alarcón-Hernández ${ }^{2}$ \\ Universidad de Concepción \\ Facultad de Humanidades y Arte \\ Biobio, Chile \\ palarco@udec.cl \\ Patricia Martínez-Ilabaca ${ }^{3}$ \\ Universidad Católica de la Santísima Concepción \\ Departamento de Lenguas \\ Biobio, Chile \\ pmartine@ucsc.cl \\ Iris Roa-Ghiselini ${ }^{4}$ \\ Universidad Católica de la Santísima Concepción \\ Departamento de Lenguas Concepción \\ Biobio, Chile \\ iroa@ucsc.cl \\ María Gabriela Sanhueza-Jara ${ }^{5}$ \\ Universidad Católica de la Santísima Concepción \\ Departamento de Lenguas Concepción \\ Biobio, Chile \\ gabrielasanhueza@ucsc.cl
}

Recibido: 27 enero 2014 Aceptado: 9 abril 2015 Corregido: 11 mayo 2015

1 Profesor de Inglés, Doctor en Educación y Magíster en Artes con mención en Lingüística por la Universidad de Concepción, Chile. Certificate in English Language Teaching to Adults (CELTA) Oxford Brookes University. Investigador responsable proyecto FONDECYT 1120247 "Investigación del conocimiento profesional, las creencias implícitas y el desempeño en aula de estudiantes de Pedagogía en Inglés como estrategia de generación de indicadores de monitoreo de su proceso formativo".

2 Profesora de Español, Doctora y Magíster en Lingüística. Departamento de Español. Universidad de Concepción.

3 Profesora de Inglés y Magíster en Educación Superior con mención en Pedagogía Universitaria. Departamento de Lenguas. Universidad Católica de la Santísima Concepción. Concepción. Chile

4 Profesora de Inglés y Magíster en Ciencias de la Educación. Departamento de Lenguas. Universidad Católica de la Santísima Concepción. Concepción. Chile.

5 Profesora de Inglés y Magíster en Ciencias de la Educación. Departamento de Lenguas. Universidad Católica de la Santísima Concepción. Concepción. Chile. 
Resumen: Como agente reflexivo de su propia práctica, el profesorado construye creencias pedagógicas sobre el proceso de enseñanza y aprendizaje y, en particular, sobre el rol que sus estudiantes deben asumir en el aprendizaje de un idioma. Este estudio tiene como objetivo identificar las creencias pedagógicas de treinta docentes de inglés de primaria, secundaria y terciaria, en Chile, sobre el rol estudiantil en el proceso didáctico. Un cuestionario, una entrevista semi-estructurada, un diario autobiográfico y cinco diarios de observación no-participante fueron utilizados para revelar las creencias pedagógicas de sus informantes. Los datos arrojados por los instrumentos fueron analizados con el programa cualitativo ATLASTI, que permitió el levantamiento de tres dimensiones y diez categorías, mediante el uso de la técnica del análisis de contenido semántico. Los resultados muestran que los tres grupos de profesorado declaran que estudiantes que aprenden una lengua extranjera deben tener características personales y académicas relacionadas con la motivación, la participación, la autonomía, entre otras; sin embargo, al interior del aula asumen un rol pasivo y reactivo frente al ambiente de enseñanza altamente estructurado que genera el personal docente. En conclusión, las creencias que cada docente sostiene se alimentan de la teoría y se corroboran o cuestionan en la práctica de aula, a modo de una relación bidireccional entre teoría y práctica, y viceversa.

Palabras clave: Creencias, estudiante, personal docente, inglés, enseñanza, aprendizaje.

\begin{abstract}
As reflective practitioners, teachers build their own pedagogical beliefs about teaching and learning and particularly about the role played by students in language learning. This study aims to identify the pedagogical beliefs held by a group of thirty Chilean primary, secondary and tertiary teachers of English about the role of students in the teaching process. A survey, a semi-structured interview, a journal and five class observation journals were employed to uncover the participants' beliefs. The data were analyzed through the qualitative data analysis program ATLASTI that led to the construction of three dimensions and ten categories, through the use of the semantic content analysis technique. The results show that the three groups of teachers declare that students who learn a foreign language should possess personal and academic characteristics related to motivation, participation and autonomy, among others; however, at the classroom those students assume a passive and reactive role towards the highly structured teaching environment created by the teacher. In conclusion, teacher beliefs are fed by theory and corroborated or questioned by classroom practice as a bidirectional relationship between theory and practice and vice versa.
\end{abstract}

Keywords: beliefs, students, teacher, English, teaching, learning.

Cada docente posee un conjunto de creencias, sean estas religiosas, ideológicas, políticas o de cualquier tipo. No obstante, cuando se aborda el proceso de enseñanza y aprendizaje, se está en presencia de creencias pedagógicas, las que han sido investigadas por su aplicación práctica en situaciones didácticas. La dimensión cognitiva de un sujeto está conformada por entramados complejos de conocimientos, creencias, actitudes, pensamientos, percepciones y representaciones, difíciles de delimitar; pero altamente influyentes en la forma de actuar de un sujeto y, específicamente, en su desempeño en el aula (Díaz y Solar, 2011; Farrell, 2006; Goodson y Numan, 2002). Este fenómeno ha sido poco explorado, a pesar de que el mundo cognitivo de docentes de lenguas puede aportar significativamente en la comprensión de sus actuaciones áulicas, sus decisiones y su aproximación al cambio y a la innovación pedagógica, procesos que los invaden por todas partes.

El estudio que se presenta a continuación se inserta en el contexto del proyecto FONDECYT 1120247: Investigación del conocimiento profesional, las creencias implícitas y 
el desempeño en aula de estudiantes de Pedagogía en Inglés como estrategia de generación de indicadores de monitoreo de su proceso formativo; y del proyecto FONIDE 09: Los procesos de cognición y actuación docente como ejes articuladores del saber pedagógico y su impacto en el diseño de un modelo de práctica pedagógica reflexiva para la formación inicial docente: Un estudio en algunos establecimientos municipalizados de la octava región.

En síntesis, mediante una metodología cualitativa, este artículo tiene como objetivo identificar las creencias pedagógicas de un grupo de treinta docentes de inglés de distintos niveles educacionales sobre el rol que, desde su perspectiva, sus estudiantes deben tener y asumir en el proceso de enseñanza y aprendizaje del idioma.

\section{Marco teórico}

En el contexto de la sociedad actual, el personal docente es considerado como un sujeto reflexivo que usa la auto-reflexión como una forma de superar los juicios y las respuestas estereotipadas. Ello significa pensar y analizar críticamente las propias acciones, con el fin de mejorar las prácticas pedagógicas (Carter y Nunan 2001; Castellanos, 2013; Crookes, 2003; Savignon, 2002; Schôn, 1998). Cuando se reflexiona en la acción misma de enseñar, quien se dedica profesionalmente a la educación se convierte en un investigador e investigadora de su práctica, libre de teorías y técnicas establecidas, y capaz de construir una nueva teoría que encaje en una situación única. Ahora bien, las presunciones y los valores que el profesorado posee sobre la enseñanza deberían ser explicitados en la lógica de un marco constructivista de enseñanza y aprendizaje en los planes de formación docente, si es que tienen como objetivo impactar la estructura profunda de conocimientos y creencias del profesorado o de estudiantes que se forman para serlo (Borg, 2003; Burns y Richards, 2009; Brown, 2001).

El concepto de creencia, para Levin y He (2008), constituye las teorías personales del cuerpo docente sobre la enseñanza y el aprendizaje. Se distinguen dos tipos de teorías personales: 1- aquel conocimiento teórico que cada docente espera usar y 2- el conocimiento teórico en la acción, constituido por aquello que las personas realmente usan en la vida real cotidiana. Todas las presunciones, reflexiones y perspectivas del profesorado constituyen su psicología de la vida diaria; son ideas que responden al sentido común, que no han sido verbalizadas y que se generan a partir de la experiencia personal (Johnstone, 2003; Kasoutas y Malamitsa, 2009; Mora, Trejo y Roux, 2014). Cualquier profesional de la educación posee teorías que guían sus prácticas (Díaz, Martínez, Roa y Sanhueza, 2010; González, Río y Rosales, 2001).

Las teorías personales sobre la enseñanza y el aprendizaje consisten en: 1) creencias centrales (afirmaciones declarativas sobre lo que se asume es verdadero, 'correcto' o 'apropiado' sobre el proceso didáctico); 2) el conocimiento fundacional, es decir, el cuerpo de conocimientos o habilidades que se consideran como esencial para la enseñanza efectiva; un 'guion' para la enseñanza, y 3) la teoría informal sobre la enseñanza que otorga un sentido de rol y responsabilidad, y una teoría sobre lo que funciona y lo que no en la enseñanza (Hearn y Garcés, 2003; Montijano, 2001; Spiro, 2013).

Tanto conocimientos como creencias no pueden ser diferenciados claramente, puesto que, como constructos psicológicos, afectivos y sociales, se entrelazan y tiñen. No obstante, lo que 
sí es evidente es que ambos inciden en la toma de decisiones de un sujeto y, finalmente, en su desempeño (McBride, 2014; Richards y Rodgers, 2001).

La investigación empírica muestra que las creencias del profesorado difieren significativamente respecto a su correspondencia con las prácticas pedagógicas cotidianas. Las creencias se reflejan en su conocimiento pedagógico de la disciplina, se muestran en las concepciones sobre los roles de docente y discente; se relacionan con el rendimiento académico estudiantil y varían ampliamente (Levin y He, 2008; Milicia, Utges, Salinas, y San José, 2004).También se evidencian diferentes grados de consistencia entre las creencias pedagógicas y las prácticas instruccionales (Kember, 2001; Kennedy, 2002). No obstante, no está tan claro, en esta relación dialéctica, si las creencias influyen en las prácticas o las prácticas influyen en las creencias.

\section{El rol estudiantil en una lengua extranjera}

Los diferentes enfoques didácticos para la enseñanza y aprendizaje de una lengua extranjera asignan roles estudiantiles distintos. Los enfoques más tradicionales, centrados en el rol activo del profesorado -como es el caso de los enfoques gramatical y audiolingualconsideran al estudiante o a la estudiante como un individuo pasivo y recipiente de la transmisión de información que entrega quien enseña. Desde esta perspectiva, el personal docente de inglés es modelo lingüístico y pedagógico de un idioma extranjero y su rol fundamental consiste en el modelamiento de las estructuras gramaticales, léxicas y fonológicas que deben aprender sus estudiantes a un nivel de dominio similar al de una persona hablante angloparlante. Los enfoques comunicativos actuales -enfoque comunicativo y centrado en tareas- ponen un fuerte énfasis en la interacción comunicativa entre los sujetos y el mensaje que intercambian, de forma tal que cada estudiante asume un rol protagónico y central en el proceso de enseñanza y aprendizaje del idioma, y el personal docente asume un rol que facilita y guía el acto didáctico. Esta delimitación tan clara desde el punto de vista teórico no necesariamente es así en la práctica mediada por las interacciones entre docentes y discentes, de ahí la importancia de conocer las creencias que sostiene el profesorado de inglés respecto del rol estudiantil en el proceso didáctico.

\section{Diseño de investigación}

\section{Objetivo de la investigación}

- Identificar las creencias lingüístico-pedagógicas de un grupo de docentes de inglés de enseñanza primaria, secundaria y terciaria sobre el rol estudiantil de quien aprende el idioma. 


\section{Tipo de estudio}

Este estudio es no experimental de tipo transeccional exploratorio, pues su objetivo es identificar las creencias lingüístico-pedagógicas de este conjunto de docentes de inglés, en Chile (Canales, 2006; Cerda, 2007). Se trata de una exploración inicial en un momento específico y acerca de un problema de investigación nuevo o poco conocido (Albert, 2007; Bisquerra, 2009). El estudio se inscribe en la metodología cualitativa del estudio de casos de tipo interpretativo (Sandín, 2003; Sevillano, Pascual y Bartolomé, 2007). Este estudio se nutre de dos investigaciones que comparten su objetivo general y enfoque metodológico, pero se ejecutan con muestras distintas. La primera de ellas aborda profesorado de inglés de la enseñanza primaria y secundaria, y la segunda de ellas involucra profesorado de inglés de la educación terciara. Ambos estudios están referenciados en la introducción de este estudio.

\section{Sujetos informantes}

Estos sujetos informantes corresponden a una muestra no-probabilística de 30 sujetos distribuidos en: 10 docentes de inglés de primaria, 10 de secundaria y 10 de educación terciaria. Fue un muestreo subjetivo por decisión razonada, es decir, las unidades de la muestra no se eligieron usando procedimientos probabilísticos, sino en función de algunas de sus características específicas, tales como:

- Ser docente de inglés formado en una universidad.

- Estar trabajando en uno de los niveles educacionales: primario, secundario o terciario.

- Participar voluntariamente en la investigación.

- Completar todos los instrumentos de la investigación.

- Poseer dos años de experiencia laboral.

\section{Supuestos del estudio}

Aunque de naturaleza compleja, el estudio de las creencias lingüístico-pedagógicas de personal docente de inglés sobre el rol de sus estudiantes en el proceso de enseñanza y aprendizaje del idioma puede contribuir a desentrañar lo que creen, hacen y saben sobre sus estudiantes.

\section{Instrumentos}

Los treinta informantes respondieron la siguiente batería de instrumentos, validados todos en su totalidad por juicio de personas expertas, y piloteados con cinco informantes que no fueron parte de esta muestra, a saber:

- Entrevista semi-estructurada en torno a ocho temas centrales relacionados con sus estudiantes. Su propósito era develar las creencias lingüístico-pedagógicas de las personas informantes respecto al rol discente en el proceso didáctico (Wilkinson y Birmingham, 2003). 
- Diario autobiográfico que las personas informantes mantuvieron por un período de seis meses, con la finalidad de consignar sus reflexiones sobre la función de sus estudiantes en el proceso didáctico en el aula.

- Cuestionario tipo LIKERT que permitió profundizar las creencias lingüísticopedagógicas que las personas informantes sostenían sobre sus estudiantes.

- Diarios de observación no participante de clases a los que fueron sometidas las personas participantes en cinco oportunidades cada una. En cada observación se consignaban los registros relacionados con la interacción que informantes sostenían con sus propios estudiantes.

- La siguiente Tabla 1 muestra algunos extractos de los instrumentos utilizados:

Tabla 1

Muestra de los instrumentos utilizados

Ítem del cuestionario

El rol fundamental del estudiantado es desarrollar una competencia avanzada en el análisis de las estructuras gramaticales y la memorización de listados de estructuras léxicas para traducir textos escritos.

\section{Ítem de la entrevista semi-estructurada}

¿De qué manera cree usted que el contexto social de sus estudiantes influye en la forma de enseñar?

Ítem del diario autobiográfico

Dibuje una línea de tiempo que represente el tiempo que lleva enseñando y marque aquellas etapas claves en su formación como profesor o profesora.

Ítem de la pauta de observación

Establece relaciones con los temas estudiados o los conocimientos previos del grupo de estudiantes

Nota: Elaboración propia.

\section{Procedimiento de análisis de los datos}

Los datos provenientes de la entrevista semi-estructurada, los diarios autobiográficos y los diarios de observación no-participantes se trataron mediante la técnica del análisis de contenido semántico, sintetizada en el siguiente tratamiento de los datos (Corbetta, 2007; Creswell, 2007):

- Transcripción, segmentación y codificación inicial de los datos mediante el uso del programa ATLASTI.

- Identificación de los segmentos relevantes de los discursos de las personas informantes.

- Establecimiento de las unidades de significado.

- Búsqueda sistemática de las propiedades de las unidades de significado. 
- Saturación de los datos.

- Triangulación de los datos cualitativos mediante las diversas técnicas utilizadas.

- Levantamiento de las categorías y subcategorías.

- Búsqueda de relaciones entre las categorías y sub-categorías.

- Elaboración de la rejilla de validación para las categorías y subcategorías mediante la triangulación por investigador que garantiza la confiabilidad en los estudios cualitativos.

Los datos provenientes del cuestionario tipo LIKERT fueron sometidos a una análisis de frecuencia simple, que permitió fortalecer los resultados del análisis cualitativo. En este artículo se presenta un análisis integrado de los datos que permita a quien lo lea obtener una fotografía de las creencias lingüístico-pedagógicas de las personas informantes.

\section{Resultados y discusión de los datos}

Los datos que se presentan a continuación se han organizado de la siguiente forma ${ }^{6}$ en la Tabla 2:

Tabla 2

Organización de los datos analizados

\begin{tabular}{lll}
\hline Dimensiones & Categorías & Subcategorías \\
\hline - Profesorado de enseñanza & $\begin{array}{l}\text {-Características del estudiantado de } \\
\text { primaria }\end{array}$ & $\begin{array}{l}\text {-Compromiso de docente } \\
\text { con estudiante }\end{array}$ \\
\cline { 2 - 2 } & -El rol estudiantil al interior del aula \\
\hline $\begin{array}{l}\text { - Profesorado de enseñanza } \\
\text { secundaria }\end{array}$ & \begin{tabular}{l}
-El rol estudiantil \\
\cline { 2 - 2 }
\end{tabular} & $\begin{array}{l}\text {-Las condiciones de vulnerabilidad del } \\
\text { estudiantado }\end{array}$ \\
\cline { 2 - 2 } & -El rol del estudiantado al interior del \\
\hline - Profesorado de enseñanza & aula \\
\hline terciaria & -Características de estudiantes con éxito \\
\cline { 2 - 2 } & $\begin{array}{l}\text {-Rol del estudiantado en el proceso de } \\
\text { enseñanza y aprendizaje del idioma }\end{array}$ \\
\cline { 2 - 2 } & -Interacciones del estudiantado \\
\cline { 2 - 2 } & $\begin{array}{l}\text {-Organización del espacio físico de la sala } \\
\text { para el trabajo estudiantil }\end{array}$ \\
\cline { 2 - 2 } & $\begin{array}{l}\text { - El rol del estudiantado universitario al } \\
\text { interior del aula }\end{array}$ \\
\hline
\end{tabular}

Nota: Elaboración propia.

6 Se utilizará la nomenclatura S más un número para indicar el número del sujeto que señaló el enunciado. Ej. S1: Sujeto 1. 


\section{Dimensión I: Profesorado de enseñanza primaria}

Categoría: Caracteristicas del estudiantado de primaria. Para este grupo de docentes, el estudiantado de enseñanza primaria debe poseer una serie de características que permitan garantizar el éxito de su aprendizaje. En este punto se evidencian dos perspectivas: Una primera visión que implica contar con estudiantes que posean rasgos de actividad, participación y motivación; y una segunda que alude a estudiantes que se caracteriza por el orden, la concentración y el método. Así, el estudiantado no solo debe poseer ciertas características personales y académicas que le permitan aprender, sino que debe además demostrarlas en clases (Northcote, 2009; Seferoglu, Korkmazgil y Ölcü, 2009). Las siguientes declaraciones de las personas informantes descritas en la Tabla 3 ponen en evidencia este punto:

Tabla 3

Declaraciones de informantes sobre las características de estudiantes

\begin{tabular}{|l|c|}
\hline \multicolumn{1}{|c|}{ Declaraciones } & Frecuencia \\
\hline $\begin{array}{l}\text { Los estudiantes más exitosos en clases de inglés son aquellos que adoptan un rol activo y } \\
\text { participativo. }\end{array}$ & 6 \\
\hline $\begin{array}{l}\text { Los estudiantes más exitosos en clases de inglés son aquellos que adoptan un rol metódico } \\
\text { y ordenado. }\end{array}$ & 5 \\
\hline $\begin{array}{l}\text { Los estudiantes más exitosos en clases de inglés son aquellos que adoptan un rol concen- } \\
\text { trado y atento. }\end{array}$ & 5 \\
\hline El trabajo independiente es el tipo de estrategia que promuevo en clases. & 4 \\
\hline Los estudiantes más exitosos en clases de inglés son aquellos que adoptan un rol motivado. & 5 \\
\hline
\end{tabular}

Nota: Elaboración propia.

Para la totalidad de informantes de enseñanza primaria, el rol estudiantil principal es su carácter negociador y participante activo en la comunicación en el idioma extranjero, lo que se demuestra en el 100\% de aprobación que otorgaron al siguiente enunciado del cuestionario:

S1: El rol principal del estudiante es ser un negociador, un individuo que se comunica en la lengua extranjera e interactúa, es decir, entrega y recibe información en contextos comunicativos diversos.

El carácter activo del estudiantado en el proceso de enseñanza y aprendizaje es promovido por los enfoques didácticos comunicativos que privilegian la interacción y la negociación de significado entre participantes por sobre el aprendizaje de las estructuras fonológicas, sintácticas y léxicas descontextualizadas, que aprendidas de forma aislada no favorecen la comunicación.

Subcategoría: Compromiso de docente con estudiante. Las personas informantes también destacan su compromiso con sus estudiantes para que, efectivamente, progresen en sus aprendizajes, como se indica en el siguiente fragmento extraído de la entrevista: 
S5: La conclusión a la que he llegado es que lo fundamental es el compromiso con los alumnos, trabajar 'duro' en el aula para que ellos tengan éxito y el compromiso con la unidad educativa trabajando en equipo para que éste tenga también éxito.

Para Crookes (2003), el aprendizaje ocurre con mayor probabilidad cuando se quiere aprender. El aprendizaje de una lengua extranjera es diferente al aprendizaje de otros contenidos, debido al carácter social de dicha tarea. El aprendizaje de un idioma implica mucho más que el simple aprendizaje de habilidades comunicativas; implica una alteración de la autoimagen, la adopción de nuevas conductas sociales y culturales, y de nuevas formas de ser, lo que produce un impacto importante en la naturaleza social del estudiantado. Aprender una lengua es aprender a ser una persona social distinta; por lo tanto, el éxito a la hora de aprender una lengua extranjera se verá influido especialmente por las actitudes que se tienen hacia la comunidad de hablantes de esa lengua (Williams y Burden, 1999).

Categoría: El rol estudiantil al interior del aula. Al observar el rol del estudiantado al interior del aula se pudo establecer que la mayoría de docentes que se observó asumen un rol protagónico, caracterizado por la excesiva apropiación del discurso en el aula la mayor parte del tiempo, lo que resta oportunidades de interacción al estudiantado. Las personas informantes, además, modelan las actividades y monitorean el trabajo de sus estudiantes regularmente. Solo un informante promueve la inferencia de léxico, el uso frecuente del idioma en el aula y la enseñanza de estrategias para la comunicación activa.

La mayoría de estudiantes no participa espontáneamente en las actividades que la persona informante genera, ya que no se esfuerzan por usar el idioma inglés ni aprovechan las instancias de interacción con sus pares o su docente, lo que refleja el rol pasivo que asumen en la clase de inglés como lengua extranjera en la enseñanza primaria. Esto impacta en el uso del idioma al interior de la sala de clases, el cual es prácticamente nulo. En definitiva, el rol activo, participativo, metódico que las personas informantes declaran sobre sus estudiantes difiere significativamente de lo que se observa en el aula, donde el estudiantado evidencia un rol pasivo, no-participativo e indiferente hacia la lengua.

Un factor que resulta necesario considerar es el hecho de que el estudiantado que aprende otra lengua difiere en ansiedad, pues quienes tienden a tener altas expectativas sobre su desempeño lingüístico tienen una alta preocupación por la opinión de los otros individuos y un alto nivel de preocupación por los errores que cometen. La ansiedad lingüística está relacionada con el perfeccionismo. Una manera de ayudar a este tipo de estudiantes es enseñándoles estrategias comunicativas que les lleven a atribuir sus éxitos al uso de estrategias y no, solamente, a la habilidad o al esfuerzo personal. Para Johnstone (2003), los sujetos ansiosos experimentan pensamientos distractores que les impiden focalizarse en la actividad y en procesos cognitivos de orden superior como la organización, la interpretación y la recuperación de información.

\section{Dimensión II: El profesorado de enseñanza secundaria}

Categoría: El rol estudiantil. Las personas informantes de enseñanza secundaria resaltan también las características individuales que estudiantes de este nivel educativo 
deberían tener. Este grupo de informantes enfatiza en su discurso los rasgos propios del estudiantado para que su aprendizaje sea exitoso por sobre la demostración de estos rasgos al interior del aula. Nadie señaló la participación en clases como una característica esencial del estudiantado. La Tabla 4 refleja esta reflexión:

Tabla 4

Declaración de informantes de secundaria sobre el rol estudiantil

Declaraciones

El rol discente debería ser activo, interesado y motivado en aprender.

Categoría: Las condiciones de vulnerabilidad del estudiantado. Una parte de las personas informantes de enseñanza secundaria establecen una analogía interesante con la película Dangerous Minds, cuyo tema central son las peripecias y aventuras que debe enfrentar una profesora en un establecimiento educacional para estudiantes de alta vulnerabilidad social. Para quienes participaron como informantes, esta película refleja su forma de enseñar y sus creencias sobre la enseñanza y el aprendizaje, puesto que han debido desarrollar estrategias de aprendizaje más atractivas para atraer la atención, motivación y participación de los grupos de estudiantes más vulnerables. A continuación el siguiente fragmento de uno de los informantes refleja este punto:

S9: La profesora de la película me representa, representa el tipo de estudiante que tengo en clase, estudiantes con problemas sociales, económicos, con muchas carencias.

Categoría: El rol estudiantil al interior del aula. Según los análisis de los datos recogidos respecto de la actuación en el aula del grupo de informantes de enseñanza secundaria, se pudo establecer que más de la mitad de docentes usan el idioma inglés en la mayor parte de sus clases. Asimismo, la mayoría recoge información mediante la apelación a los conocimientos y experiencias previas de sus estudiantes y al modelamiento de las actividades que les presentan; promueven la deducción del significado de léxico sin poner mayor énfasis en la memorización. Regularmente monitorean el trabajo de sus estudiantes al interior del aula. Sin embargo, no se observa la promoción de interacciones comunicativas entre estudiantes. Solo la mitad de los informantes promueve alguna estrategia para desarrollar la comunicación en el idioma extranjero. En general, casi la totalidad incorpora actividades de repetición de aspectos fonológicos, al parecer buscando la precisión lingüística en la reproducción de discursos orales.

Se puede apreciar que casi la totalidad de estudiantes no participa de manera espontánea en las actividades llevadas a cabo en clases. En general, se puede identificar que no asumen un rol activo en términos de gestionar su propio aprendizaje o de aprovechar la clase como una instancia de práctica espontánea del idioma. Se limitan a completar de forma mecánica las actividades entregadas por el personal docente, lo que hace que su participación sea más bien reactiva. 
Al respecto, se puede señalar que el aporte fundamental de un enfoque didáctico comunicativo es ofrecer a estudiantes una práctica que les conduzca directamente a implicarse en intercambios comunicativos de diversa índole. A partir de este supuesto, lo que realmente interesa en el aula de lengua extranjera es la actuación directa del estudiantado mediante la lengua meta, el logro de diversas funciones comunicativas, al tiempo que se les presta atención a las relaciones entre las diversas oraciones que componen su discurso, los contextos y las situaciones en que aparecen.

\section{Dimensión III: Los profesores de enseñanza terciaria}

Categoría: Características del estudiantado exitoso. Al momento de señalar las características discentes que influían en su éxito durante el proceso de aprendizaje del idioma, los más mencionados fueron los aspectos relacionados con las disposiciones, tales como: la responsabilidad, el esfuerzo, la participación en clases, la constancia o la motivación, entre otros. El segundo lugar es compartido por el rendimiento académico previo de los estudiantes y ciertas características cognitivas como: la habilidad para tomar apuntes de calidad, la habilidad de escribir o leer y la aptitud para aprender idiomas, como lo muestran los siguientes fragmentos.

S20: Yo creo que va de la mano lo cognitivo con lo actitudinal porque a aquellos alumnos que son más respetuosos, cumplidores, responsables, que preguntan y participan, les va mejor.

S27: Les va mejor a los alumnos que llegan del nivel socioeconómico medio hacia arriba... a los que tienen hábitos de estudio adquiridos, a esos les va mucho mejor.

S21: [Les va mejora a aquellos estudiantes] que tienen una actitud positiva hacia la participación. Los que no tiene vergüenza de equivocarse... de participar, sobretodo. [A] los que tienen autonomía [les va bien] porque yo siempre trabajo para desarrollar esa parte, pero si uno no está dispuesto a hacer las cosas por su cuenta, va a tener dificultades.

La figura 1 sintetiza las declaraciones de informantes sobre las características de discentes con éxito. 
Categoría Características de discentes con éxito

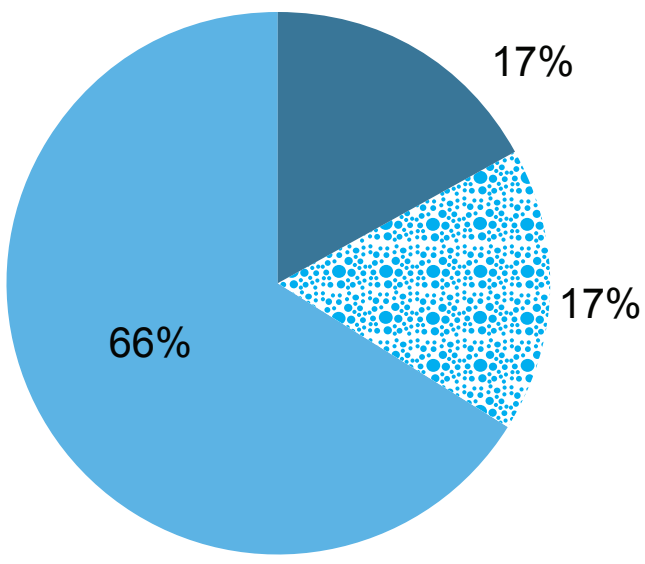

6. Características

6.: cognitivas

Cognitivas

Características

actitudinales

Rendimiento

académico previo

Figura 1. Características de discentes de universidad con éxito. Elaboración propia.

Al respecto, la didáctica comunicativa del inglés surge en oposición a la didáctica conductista. La didáctica comunicativa centra su atención en la enseñanza y el aprendizaje de la comunicación como un acto creativo, consciente, espontáneo, que se produce entre dos o más sujetos activos, donde la forma lingüística y el significado forman una unidad dialéctica indisoluble. La categoría rectora es la función comunicativa, es decir, la manifestación de las necesidades de los sujetos interactuantes para emitir opiniones, solicitar o proporcionar información, invitar a alguien, etc. Las funciones comunicativas determinan la forma lingüística a utilizar y no viceversa (Montijano, 2001). Tanto la organización curricular como las actividades docentes deben seguir la estrategia función-forma lingüística, partiendo de la primera y sin obviar la segunda, aunque no de una manera absoluta y rígida. Desde esta concepción, la lengua opera en el proceso didáctico con propósitos comunicativos bien definidos.

\section{Categoría: Rol del estudiantado en el proceso de enseñanza y aprendizaje del} idioma. Esta categoría permite evidenciar que la totalidad de los informantes declara, principalmente en las entrevistas semi-estructuradas, que el rol del estudiante durante sus clases es activo, autónomo y participativo. Sin embargo, parece interesante mencionar que la información obtenida desde los diarios autobiográficos no concuerda con estas declaraciones. En los diarios autobiográficos se aprecia una alta tendencia al control y protagonismo por parte de los profesores; además de un bajo nivel de participación activa por parte de los estudiantes. Esta aparente contradicción podría indicar que el diario autobiográfico, a diferencia de la entrevista, recoge con mayor precisión las concepciones y las tensiones en los discursos de los informantes, puesto que el diario es un instrumento que el informante completa al finalizar su clase, lo que favorece y potencia las habilidades reflexivas de los informantes. A continuación se presenta el siguiente fragmento de la entrevista: 
S29: "[Los estudiantes son] muy participativos, muy activos. [Es necesario] que no sean tan pasivos; que hablen, que conversen, que sigan instrucciones, que trabajen mucho en grupos, que intercambien información, que hagan muchas preguntas."

Existen dos binomios fundamentales en el proceso de enseñanza y aprendizaje de otra lengua: primero, el binomio humano, constituido por la personalidad del docente y la de sus estudiantes en interacción activa y continua: El segundo es el binomio cultural, formado por el contenido, las actividades y la metodología didáctica, al servicio de los agentes del binomio humano y en función de los objetivos que éstos se proponen. Será siempre una distorsión el atribuir énfasis exagerados al contenido, a las actividades o a la metodología, como si fueran datos únicos o decisivos del proceso de enseñanza y aprendizaje; en realidad, las variables del binomio cultural desempeñan en el plano pedagógico la función, necesaria pero auxiliar e instrumental para el proceso didáctico. Nunca se debe perder de vista a los estudiantes, a quienes, por encima de todo, se debe ayudar, incentivándolos y brindándoles orientación y oportunidades comunicativas significativas (Montijano, 2001).

Categoría: Interacciones de los estudiantes. Respecto a las interacciones utilizadas con mayor frecuencia, el profesorado señala, principalmente, el trabajo en pares. Asimismo las personas informantes manifiestan trabajar una combinación de interacciones en el aula, dependiendo de las distintas etapas de la clase. La mayoría de informantes manifiesta que la interacción más frecuente es entre docente y estudiantes. Un grupo menor señala utilizar el trabajo en grupo. La principal causa de esta baja frecuencia es que consideran que el estudiantado tiende a distraerse y no realiza las actividades que se le asignan, cuando trabajan en grupo. A continuación se observan algunos fragmentos que ilustran esta afirmación.

S20: Yo creo que todas [las interacciones ocurren en clases], porque les hago harto trabajo en grupos, en pares e incluso tengo como bien delimitado a los estudiantes que son más avanzados, que saben más y ellos siempre tienen muy buena disposición para ayudar al resto, entonces los dejo de monitores.

S21: La interacción era en grupos pequeños, realizando tareas distintas, y la interacción de los grupos con el profesor.

La figura 2 sintetiza los tipos de interacciones que, según declaran las personas informantes, ocurren con mayor frecuencia en la sala de clases. 
Categoría: Interacciones más frecuentes durante las clases

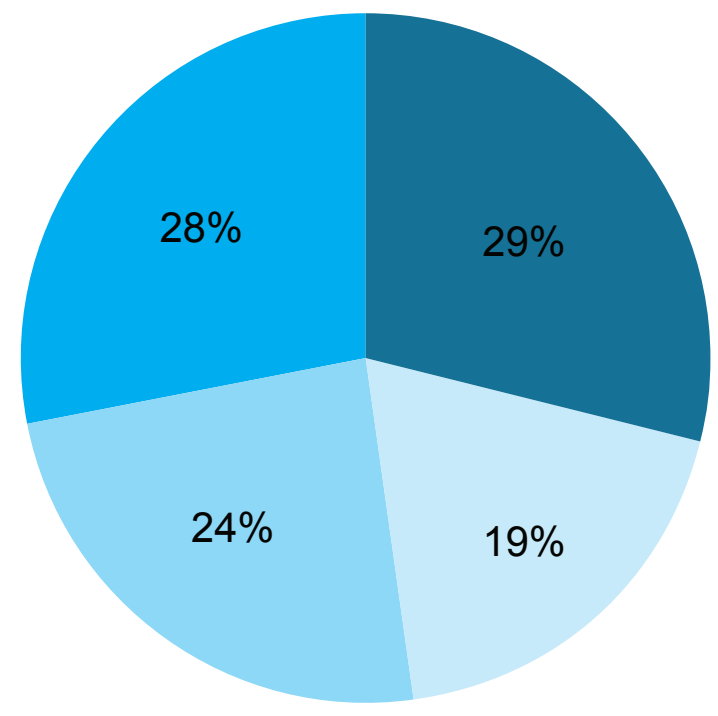

Trabajo en grupo

Docente-estudiante

Estudiante-estudiante

Conjunto de

interacciones

Figura 2. Interacciones más frecuentes en el aula universitaria. Elaboración propia.

Categoría: Organización del espacio físico de la sala para el trabajo de sus estudiantes. En cuanto a la forma en que las personas informantes organizan el aula para trabajar con sus estudiantes, la mayoría declaró organizarla en semicírculo, con el fin de fomentar la comunicación. Otro grupo de informantes prefiere la distribución en grupos pequeños, con la finalidad de que todo el estudiantado participe activamente durante la clase; por otro lado, un tercer grupo de informantes manifiesta mantener la distribución que tiene el aula en la que le corresponde realizar su clase, sin hacer cambios. Se presentan algunos fragmentos que ilustran lo expuesto.

S22: Dependiendo el inmobiliario de la universidad trato que [los estudiantes se organicen en] semi-círculo, lo ideal es que todos se vean de una vez, de repente en grupo.

S24: Se presenta un power point con personajes y ellos trabajan en grupos utilizando verbos dados en el tiempo correspondiente.

S28: Se agrupó a los alumnos en pares, grupos y trabajo individual.

La figura 3 sintetiza la forma en que declaran organizar el aula para sus clases. 


\section{Categoría: Organización del aula}
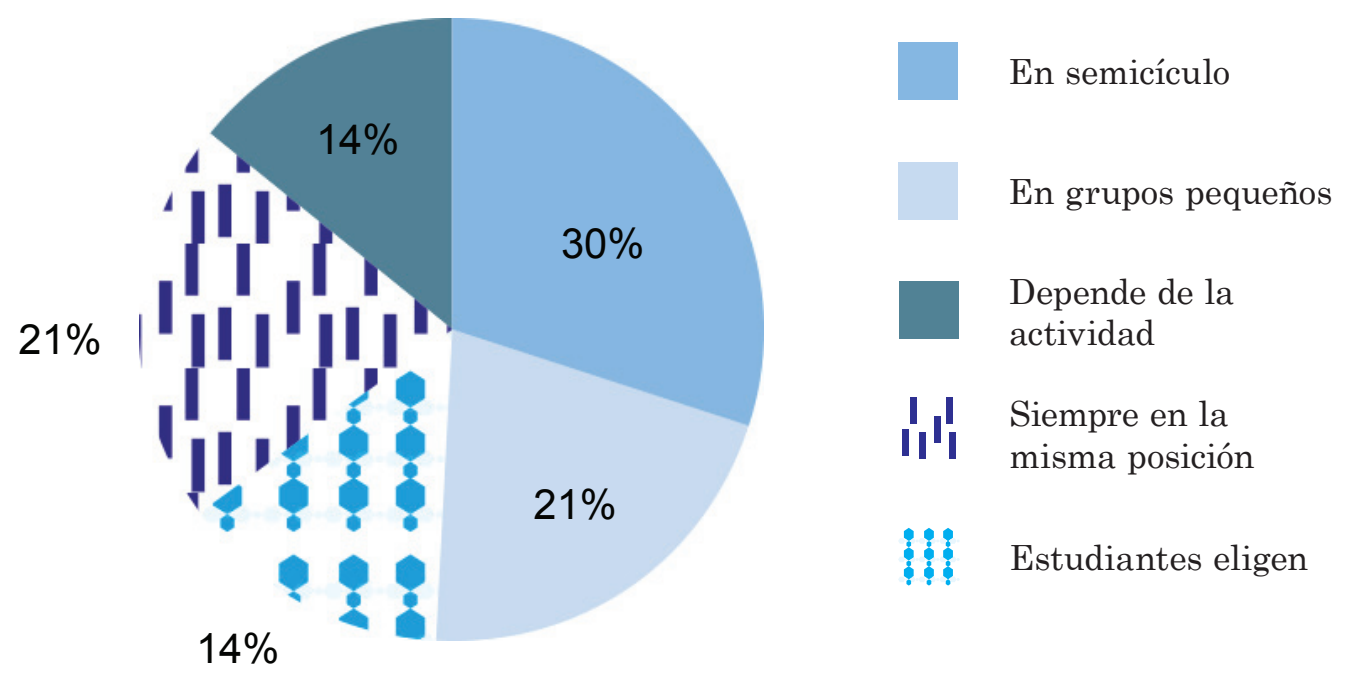

Figura 3. Organización del aula universitaria. Elaboración propia.

Categoría: El rol del estudiantado universitario al interior del aula. Desde el punto de vista del discurso, la mayoría de informantes declaró que el rol estudiantil en su clase se condice con el modelo comunicativo de la lengua inglesa, que promueve al estudiante y la estudiante como un sujeto autónomo y capaz de tomar decisiones, independiente. Esto hace que teóricamente las personas informantes crean firmemente que ese es el tipo de estudiante que se quiere formar en la universidad. Sin embargo, en la sala de clase, y a través de la pauta de observación, se constató que el estudiantado es más bien pasivo en su rol. Solo ejecutan lo que se les indica, se observan pocos momentos donde la interacción les permite comunicarse entre sus pares o inclusive con su docente.

En el aprendizaje de otra lengua, el trabajo grupal de estudiantes es muy importante, debido a que posibilita el intercambio de información entre participantes. Generalmente, se crean distintos contextos sociales, en los que los grupos de estudiantes desarrollan la lengua meta. El personal docente debe ser capaz de hablar fluidamente y su función es facilitar la comunicación, más que corregir errores.

\section{Conclusiones}

Las creencias pedagógicas que construye el personal docente de inglés de los tres niveles educacionales responden a sus teorías personales que se han ido formando como resultado de su propia escolaridad, sus experiencias profesionales y su contacto cotidiano con la práctica pedagógica al interior del aula. En este sentido, se observan dos tipos de creencias pedagógicas en los sujetos de este estudio: aquellas que cada docente construye autónomamente como 
producto de su bagaje social, cultural y educativo, y aquellas creencias que se tensionan, cuestionan y reformulan constantemente al interior del aula como resultado de la interacción con 'otros individuos', en este caso: sus estudiantes. Lo anterior guarda similitud con la que plantea Schön (1998) sobre la reflexión de la acción, la reflexión en la acción y la reflexión sobre la acción, como tres instancias de generación de creencias y conocimientos que ocurren antes, durante y después del proceso de enseñanza y aprendizaje. Esto tiene serias implicancias para los programas de formación docente, los cuales necesariamente deberían enfatizar el rol reflexivo de quienes se dedican a la profesión educativa cuando planifican su enseñanza (antes), la ejecutan (durante) y la reflexionan (después).

Las creencias pedagógicas de quienes fungieron como informantes de este estudio enfatizaban, en todo momento, la esperanza de que sus estudiantes tuvieran un rol activo, participativo y motivado en el proceso de enseñanza y aprendizaje. No obstante, la observación de clases evidenció que el estudiantado de los tres niveles educacionales muestra más bien un rol pasivo y no-participativo. Esto pone en relieve la necesidad de proveer perfeccionamiento para que el profesorado no solo mejore su conocimiento didáctico de la disciplina, sino que aprenda estrategias que le permitan, al interior del aula, ejercer la docencia con efectividad.

Los roles que los profesores y profesoras de este estudio asignan a sus estudiantes se relacionan con los principios comunicativos de la enseñanza de idiomas, en tanto, sus discursos evidencian las características personales y académicas que deberían tener quienes aprenden una lengua extranjera: motivación, participación, autonomía, responsabilidad, compromiso, entre otras. No obstante, en las prácticas de aula, se observa que sus estudiantes desempeñan un rol más bien pasivo y reactivo frente a un ambiente de enseñanza altamente estructurado y controlado, fundamentalmente por las interacciones docentes.

Este es un punto central que destaca la investigación sobre creencias y concepciones, respecto a que docentes experimentan 'tensiones' entre su discurso y su actuación, puesto que al develar sus creencias se sitúan desde escenarios ideales de enseñanza; no obstante, el aula constituye un ambiente altamente complejo caracterizado por un conjunto de interacciones e intervenciones bidireccionales entre docente y estudiantes, y estudiantes en su conjunto. Desde esta perspectiva, el profesorado de este estudio se ve enfrentado, en el aula, a un proceso constante de toma de decisiones, que muchas veces contradice sus propias creencias y les lleva a actuar frente a lo inesperado.

En este sentido, la conclusión fundamental de este tipo de estudios es que la teoría es una fuente importante de generación de creencias para el profesorado; sin embargo, la práctica pedagógica también lo es: se generan creencias que van desde la teoría a la práctica y otras que desde la práctica cuestionan la teoría.

Cobra, finalmente, mucho sentido que al interior de los programas de formación de docentes se transparenten las creencias pedagógicas que el estudiantado de pedagogía trae consigo desde la enseñanza secundaria, se monitoreen las creencias que va desarrollando en su formación y se identifiquen las creencias con las que egresa. Este proceso de explicitación de creencias no termina acá, sino que debe continuar durante la vida profesional de un profesor o profesora, como un proceso de aprendizaje reflexivo y continuo de 'aprender' y 'desaprender'. 


\section{Limitaciones del estudio y proyecciones}

Los estudios cualitativos son siempre cuestionados por abordar muestras que no son representativas del universo, en este caso, de docentes de inglés en Chile. No obstante, es siempre necesario reiterar que los estudios de naturaleza cualitativa abordan la complejidad y dinámica del objeto de estudio en profundidad y desde la subjetividad de los sujetos. Por lo tanto, cualquier extrapolación de los resultados de este estudio es responsabilidad del lector o lectora que considera que su realidad comparte características similares que permiten que los resultados en cuestión sean aplicables a su propio contexto.

Para una mejor profundización del tema en investigaciones futuras, podría realizarse un estudio de naturaleza mixta que permita organizar las dimensiones, categorías y subcategorías como posibles categorías de un cuestionario que recoja las creencias del profesorado de inglés de distintas regiones geográficas del país para, de tal forma, comparar si las creencias de los sujetos respecto de sus estudiantes se ven influidas por esta variable geográfica.

\section{Referencias}

Albert, M. (2007). La investigación educativa. Madrid: McGraw-Hill.

Bisquerra, R. (2009). Metodología de la investigación educativa. Madrid: La Muralla.

Borg, S. (Abril, 2003). Teacher cognition in language teaching: a review of research on what language teachers think, know, believe, and do. [La cognición docente en la enseñanza de lenguas: Una revisión de la investigación sobre lo que los profesores, piensan, conocen, creen y hacen] Language Teaching, 36(2), 81-109. doi: http://dx.doi.org/10.1017/ s0261444803001903

Burns, A. y Richards, J. (Eds.). (2009). The Cambridge Guide to Second Language Teacher Education. [La guía Cambridge sobre la formación de profesores de segundas lenguas] New York: Cambridge University Press.

Brown, D. (2001). Teaching by principles. An interactive approach to language pedagogy. [Enseñar según principios. Un enfoque interactivo para la pedagogía de lenguas] New York: Pearson Longman.

Canales, M. (2006). Metodologías de investigación social. Introducción a los oficios. Santiago, de Chile: Editorial LOM.

Carter, R. y Nunan, D. (Eds.). (2001). Teaching English to speakers of other languages. [La enseñanza del inglés a hablantes de otras lenguas] Cambridge: Cambridge University Press. doi: http://dx.doi.org/10.1017/CBO9780511667206 
Castellanos, J. (Abril, 2013). The role of English preservice teachers' beliefs about teaching in teacher education programs. [El rol de candidatos a profesores de inglés sobre la enseñanza en la formación de profesores] Profile Journal, 15(1), 195-206. Recuperado de http://www.revistas.unal.edu.co/index.php/profile/article/view/37878/40129

Cerda, H. (2007). La investigación formativa en el aula. La pedagogía como investigación. Bogota: Cooperativa Editorial Magisterio.

Corbetta, P. (2007). Metodología y técnicas de investigación social. Madrid: McGraw Hill.

Creswell, J. (2007). Qualitative inquiry and research design. Choosing among five approaches. [Diseños investigativos y aproximación cualitativa. Selección de cinco enfoques] London: Sage.

Crookes, G. (2003). A practicum in Tesol. Professional development through teaching practice. [La práctica Tesol. Desarrollo profesional mediante la práctica docente] Cambridge: Cambridge Language Education.

Díaz, C., Martínez, P., Roa, I. y Sanhueza, M. (2010). Una fotografía de las cogniciones de un grupo de docentes de inglés de secundaria acerca de la enseñanza y aprendizaje del idioma en establecimientos educacionales públicos de Chile. Revista Folios, 45(3), 69-80. Recuperado de http://www.scielo.org.co/pdf/folios/n31/n31a05

Díaz, C. y Solar, M. (2011). La revelación de las creencias lingüístico-pedagógicas a partir del discurso del profesor de inglés universitario. Revista RLA, 49(2), 57-86. doi: http://dx.doi. org/10.4067/S0718-48832011000200004

Farrell, T. (2006). The teacher is an octopus: uncovering preservice English language teachers' prior beliefs through metaphor analysis. [El profesor es un pulpo. Descubriendo las creencias previas de estudiantes de pedagogía mediante el análisis metafórico] Regional Language Centre Journal,37(2), 236-248. doi:http://dx.doi.org/10.1177/0033688206067430

González, S., Río, E. y Rosales, S. (2001). El currículum oculto en la escuela. Buenos Aires: Lumen Humanitas.

Goodson, I. y Numan, U. (2002). Teacher's life worlds, agency and policy contexts. [El mundo, las acciones y los contextos políticos de la vida del profesor] Teachers and Thinking: Theory and Practice, 8(81), 57-69. doi: http://dx.doi.org/10.1080/135406002100000422

Hearn, I. y Garcés, A. (2003). Didáctica del inglés. Madrid: Pearson Practice Hall. 
Johnstone, R. (2003). Annual review of research. Language teaching. [Revisión anual de la investigación. Enseñanza de lenguas] The International Abstracting Journal for Language Teachers and Applied Linguists, 36(2), 165-189. doi: http://dx.doi.org/10.1017/ s0261444803001976

Kasoutas, M. y Malamitsa, K. (2009). Exploring Greek teachers' beliefs using metaphors. [La exploración de las creencias de profesores griegos mediante la metáfora] Australian Journal of Teacher Education, 34(2), 64-83.

Kember, D. (2001). Beliefs about knowledge and the process of teaching and learning as a factor in adjusting to study in higher education. [Las creencias acerca del conocimiento y el proceso de enseñanza y aprendizaje como factores de ajuste para el estudio en la educación superior] Studies in Higher Education, 26(1), 205-221. doi: http://dx.doi. org/10.1080/03075070120052116

Kennedy,M.(2002). Knowledgeand teaching. [Conocimientoy enseñanza] Teachers and Thinking: Theory and Practice, 8(2), 355-370. doi: http://dx.doi.org/10.1080/135406002100000495

Levin B. y He, Y. (Enero-febrero, 2008). Investigating the content and sources of teacher candidates' personal practical theories (PPTS). [La investigación del contenido y las fuentes de las teorías personales de los candidatos a profesor] Journal of Teacher Education, 59(1), 55-68. doi: http://dx.doi.org/10.1177/0022487107310749

McBride, K. (2014). Percepciones estudiantiles sobre las técnicas utilizadas en la enseñanza del inglés como lengua extranjera. Revista Universium, 24(2), 129-144. Recuperado de http://www.scielo.cl/scielo.php?pid=S071823762009000200006\&script=sci_arttext

Milicia, B., Utges, G., Salinas, B. y San José, V. (2004). Creencias, concepciones y enseñanza en la universidad: Un estudio de caso de desarrollo profesional colaborativo centrado en un profesor de física. Revista Española de Pedagogía, 229(2), 377-394.

Montijano, M. (2001). Claves didácticas para la enseñanza de la lengua extranjera. Málaga: Aljibe.

Mora A., Trejo, P. y Roux, R. (2014). English language teachers' professional development and identities. [Las identidades y el desarrollo profesional de profesores de inglés] Profile Journal. Issues in Teachers' Professional Development, 16(1), 49-62. doi: http://dx.doi. org/10.15446/profile.v16n1.38153 
Northcote, M. (2009). Educational beliefs of higher education teachers and students: implications for teacher education. [Las creencias educacionales de profesores y estudiantes universitarios: Implicancias para la formación de profesores] Australian Journal of Teacher Education, 34(2), 69-81. doi: http://dx.doi.org/10.14221/ajte.2009v34n3.3

Richards, J. y Rodgers, T. (2001). Approaches and methods in language teaching. [Enfoques y métodos en la enseñanza de lenguas] Cambridge: Cambridge University Press. doi: http://dx.doi.org/10.1017/CBO9780511667305

Sandín, M. (2003). Investigación cualitativa en educación. Fundamentos y tradiciones. Madrid: McGraw-Hill.

Savignon, S. (2002). Interpreting communicative language teaching. Contexts and concerns in teacher education. [La interpretación de la enseñanza comunicativa de lenguas. Contextos y preocupaciones en la formación de profesores] New Haven and London: Yale University Press.

Schön, D. (1998). El profesional reflexivo. ¿Cómo piensan los profesionales cuando actúan? Barcelona: Editorial Paidós.

Seferoglu, G., Korkmazgil, S. y Ölcü, Z. (2009). Gaining insights into teachers' ways of thinking via metaphors. [Una introspección de las formas de pensamiento de los profesores mediante la metáfora] Educational Studies, 35(2), 323-335. doi: http://dx.doi. org/10.1080/03055690802648135

Sevillano, M., Pascual, M. y Bartolomé, D. (2007). Investigar para innovar la enseñanza. Madrid: Pearson. Prentice Hall.

Spiro, J. (2013). Changing methodologies in Tesol. [Cambios de la metodología Tesol] Edinburgh: Edinburgh University Press.

Wilkinson, D. y Birmingham, P. (2003). Using research instruments. A guide for researchers. [El uso de instrumentos de investigación. Una guía para los investigadores] New York: Routledge Farmer.

Williams, M. y Burden, R. (1997). Psychology for language teachers. [Psicología para los profesores de lenguas]. Cambridge: Cambridge University Press. 